\title{
An occupant-differentiated, higher-order Markov Chain method for prediction of domestic occupancy
}

\author{
Graeme Flett*, Nick Kelly \\ Energy Systems Research Unit (ESRU), Department of Mechanical and Aerospace Engineering, University of Strathclyde, Glasgow, UK
}

\begin{abstract}
Household energy demand is closely correlated with occupant and household types and their associated occupancy patterns. Existing occupancy model performance has been limited by a lack of occupant differentiation, poor occupancy duration estimation, and ignoring typical occupancy interactions between related individuals. A Markov-Chain based method for generating realistic occupancy profiles has been developed that aims to improve accuracy in each of these areas to provide a foundation for future energy demand modelling and to allow the occupancy-driven impact to be determined. Transition probability data has been compiled for multiple occupant, household, and day types from UK Time-Use Survey data to account for typical behavioural differences. A higher-order method incorporating ranges of occupancy state durations has been used to improve duration prediction. Typical occupant interactions have been captured by combining couples and parents as single entities and linking parent and child occupancy directly. Significant improvement in occupancy prediction is shown for the differentiated occupant and occupant interaction methods. The higher-order Markov method is shown to perform better than an equivalent higher-order 'event'-based approach. The benefit of the higher-order method compared to a first-order Markov model is less significant and would benefit from more comprehensive occupancy data for an objective comparison.
\end{abstract}

Keywords: occupancy, markov chain, domestic, modelling, energy demand, microgeneration, distributed generation, higher-order

\section{Introduction}

Technical and commercial analysis of distributed generation energy projects, particularly for smaller schemes with typically fewer than 500 households, requires a detailed understanding of the likely demand profile at both short and long time-scales. Intra-day demand diversity estimation is required to assess the sizing of localised energy supply systems, the demand management potential, and the scope for grid import/export.

Demand prediction is of particular importance for smallscale low-carbon projects. Generation may either be seasonal, intermittent or benefit from stable demand [1], and as the scale reduces individual household demand behaviours become increasingly influential. Accurate matching of supply and demand and adequate storage sizing are therefore critical for ensuring that such projects perform as anticipated. The UK Government has identified a lack of energy demand data as a key barrier to growth in low-carbon community energy and demand management projects [5].

\subsection{Relationship between Household Characteristics, Occu- pancy, and Demand}

The relationship between household characteristics, occupancy and demand is complex.

A number of factors have been shown from analysis of measured data to influence household energy demand characteristics. Yohanis [20], Haldi and Robinson [7], and McLoughlin

${ }^{*}$ Corresponding author. E-mail address: graeme.flett@strath.ac.uk (G.Flett) et al [8] have determined that these include, but are not limited to; floor area, household size, bedroom number, occupant age, income, social class, children, employment status, and tenure.

The specific influence of occupancy probability has also been identified. Capasso [3] incorporated occupancy potential as a primary demand driver in a developed demand model that combined a variety of socio-economic and behavioural factors. Yao and Steemers [19] concluded that "both behavioral determinants and physical determinants related energy-consumption are more or less influenced by people's occupancy pattern", and that employment related daytime absences were the most significant occupancy effect. An extensive review of the literature linking time-use behaviour and electrical demand was performed by Torriti [15], stating that "residential electricity demand profiles are highly correlated with timing of active occupancy, i.e. when consumers are at home and awake".

The link between household characteristics and occupancy was analysed in detail by Wilke [18] using French Time Use Survey data. Specific variations were observed based on employment, gender and day type (weekend/weekday) with age ranges also identified as a key factor for the developed occupancy model.

Despite the existing work linking both household characteristics and occupancy with demand. There has been little work done that specifically quantifies the impact of occupancy on demand and the related influence of different types of occupants (e.g. full-time workers, stay-at-home parents, retired individuals etc.). 
The need for research in this area becomes more critical when considering the changing demand characteristics of dwellings; as the thermal efficiency of dwellings improves, occupancy driven electrical and hot water demands will predominate as heating demand, which is less occupancy sensitive, falls. Moreover, the residual heating load in low-carbon houses may be more closely linked to active occupancy as pre-heat times reduce and heating times tend towards actively occupied periods for a proportion of potential heating system and building thermal design combinations. Consequently, realistic predictions of occupancy patterns will be crucial in determining the characteristics of future domestic energy consumption.

\subsection{Occupancy Data Sources}

There is currently no large UK dataset that specifically tracks the occupancy of individuals over a prolonged period of time. Assessing long-term occupancy patterns for individual households is therefore difficult. However, there is extensive single day time-use data, allowing assessment of occupancy patterns across identifiable sub-populations.

The UK Time-Use Survey (TUS) dataset compiled in 2000/2001 [11] was used for the initial analysis and final model development for the work reported in this paper. The dataset comprises approximately 20,000 diaries with a 10-minute resolution, with one weekday and one weekend day diary per person. Additionally a smaller UK time-use dataset was compiled in 2005 [12]. This comprises approximately 5000 diaries and includes the same data as the earlier, larger TUS survey. This later dataset was used for verification of the model outputs.

Each individual diary includes detailed personal information (age, gender, relationships to other occupants etc.), household information (size, type, age of youngest child etc.), and a primary activity, secondary activity (e.g. watching TV while undertaking primary activity) and location for each of the 144 10-minute time-steps. 146 standard TUS activities are defined that consolidate all potential occupant activities into appropriately linked groups. For example, the 'Food Prep' TUS activity comprises all cooking and meal preparation activities. The 2000/2001 survey also includes one-week work diaries from which typical working patterns can be derived.

Torriti [16] reviewed time-use datasets, identifying some inherent problems; (1) large datasets are required to provide sufficiently representative behavioural data, (2) typical days are captured, ignoring the potential for extreme weather or communal events, and (3) TUS surveys are rarely undertaken, consequently the use of older survey data for use in future projections could yield potentially misleading results. Further, the 24-hour duration of TUS diaries prevents identification of occupancy and activity patterns for individuals occurring over time periods exceeding 24 hours.

Despite these limitations, TUS datasets remains the sole source for occupancy and activity data with a sufficient breadth of respondents to be representative of the overall population and also smaller sub-populations. With a 10-minute time resolution, they provide sufficient data to allow effective modelling of occupancy and behaviour that affects energy use.

It should be noted that a new UK survey is to be completed in 2015 [4]. This dataset may show significant changes in daily activities and the work reported in this paper will be updated when this data becomes publicly available.

\subsection{Prediction of Occupancy for Demand Modelling}

Grandjean et al [6] conducted a comprehensive review of demand modelling and concluded that bottom-up models featuring stochastic occupancy prediction represented the best current method. Richardson et al [13] and Widen et al [17] have developed such models. These authors use a first-order MarkovChain approach to predict changes in occupancy.

Markov-Chain (MC) techniques allow the occupancy status at a time, $t$, to be determined based only on the status at the previous time, $t-\Delta t$. The basis for any MC model is transition matrices (see Figure 1). These hold the probability of transition from one state $a$ to another state $b\left(\mathrm{p}_{a \rightarrow b}\right)$. The size of this matrix is determined by the number of independent states to be modelled. For a model with $n$ states, an $n \times n$ matrix is required. A row in this matrix therefore contains the probabilities of a transition from some state $i$ to all $n$ possible states (including no change from state $i$ ) and all entries per row should sum to 1 .

\begin{tabular}{c|cccccc} 
State & 1 & 2 &.. & $i$ &.. & $n$ \\
\hline 1 & $\mathrm{p}_{1 \rightarrow 1}$ & $\mathrm{p}_{1 \rightarrow 2}$ &.. & $\mathrm{p}_{1 \rightarrow i}$ &.. & $\mathrm{p}_{1 \rightarrow n}$ \\
2 & $\mathrm{p}_{2 \rightarrow 1}$ & $\mathrm{p}_{2 \rightarrow 2}$ &.. & $\mathrm{p}_{2 \rightarrow i}$ &.. & $\mathrm{p}_{2 \rightarrow n}$ \\
.. &.. &.. &.. &.. &.. &.. \\
$i$ & $\mathrm{p}_{i \rightarrow 1}$ & $\mathrm{p}_{i \rightarrow 2}$ &.. & $\mathrm{p}_{i \rightarrow i}$ &.. & $\mathrm{p}_{i \rightarrow n}$ \\
.. &.. &.. &.. &.. &.. &.. \\
$n$ & $\mathrm{p}_{n \rightarrow 1}$ & $\mathrm{p}_{n \rightarrow 2}$ &.. & $\mathrm{p}_{n \rightarrow i}$ &.. & $\mathrm{p}_{n \rightarrow n}$
\end{tabular}

Fig. 1. Transition probability matrix (TPM) structure.

To calculate a sequence of states over a number of time steps, a random number $R$ between 0 and 1 is generated for each modelled time step and the new state is determined by systematically comparing the generated random number with the cumulative probabilities, $1 \ldots n$, in the appropriate row $i$ of the matrix. For example, if a state $i$ persists at time step $t$ - $\Delta t$ then $k$, the next state at time $t$, is the first cumulative probability $\sum_{j=1}^{j=k} p_{i \rightarrow j}$ that exceeds $R$.

For a first-order MC model, only the state at the preceding time step is considered. A second-order model considers the two preceding states. Higher-order models consider the duration of the existing state at each modelled time step.

In the Richardson et al [13] model, the states in the transition probability matrices (TPM) are the number of active occupants in a household, ranging from 0 to $h$, with $h$ being the total number of occupants. Consequently, different sized matrices are required for different household sizes: 2 x 2 for a 1-person household (the occupant is out or in the dwelling), $3 \times 3$ for a 2-person household (both out, one person, or two people in dwelling), etc. Widen et al [17] model each individual independently with three potential occupancy states (inactive (sleep), active, out) requiring a $3 \times 3$ matrix.

TPMs were generated for each timestep (10-minute basis for [13] and 1-minute for [17]) during the day to account for changing occupancy behaviour with time. Further differentiation is 
also made between weekdays and weekends. Therefore, depending on the current occupancy state, day type, time period, and, in the case of [13], household size, the corresponding TPM is selected to generate the next occupancy state.

\subsection{Markov Modelling Deficiencies}

Existing Markov-based methods for occupancy prediction have potential deficiencies that need to be addressed to improve occupancy modelling. First, most do not differentiate between household types beyond the number of occupants. Second, the simple, first-order approaches used do not account for the duration of a particular occupancy state [18]. This is important as certain activities (e.g. sleep, working absences) are associated with particular ranges of duration, but also with variable start and finish times that can conflict in a first-order model. Finally, occupancy interactions between different household members are not captured if modelled as multiple independent individuals [16].

\subsubsection{Differentiated Occupant Models}

Time Use Survey (TUS) data analysis highlights distinct occupancy variations for different occupant and household types, as demonstrated by Figure 2. The active occupancy probability shown is the probability of at least one occupant being awake and in the dwelling. This shows that some level of occupant or household type differentiation is required to properly capture different occupancy behaviours.

Neither the Richardson et al [13] nor Widen et al [17] models make any differentiation by household type beyond number of occupants. This implicitly assumes that different households conform to an average occupancy behaviour, which Figure 2 demonstrates is not the case. So, at the individual household level, these methods can generate occupancy profiles that are an unrepresentative composite of multiple distinct behaviours.

Further work using the same basic first-order Markov technique by Muratori et al [9] split households into four archetypes (working/non-working, male/female). Nijhuis et al [10] also used a first-order Markov method differentiated by household size and age. Neither includes a detailed analysis of the specific influence of the differentiating characteristics.

Wilke [18] reviewed the impact of sub-population type and size, including differentiation by household type, age, employment status, and gender, using an 'event'-based model that will be discussed in more detail below. He found that despite having a smaller sample size, the more refined models better replicated a particular sub-population's characteristics compared to a model derived from a larger, general population.

Aerts et al [2], using a modelling method similar to Wilke [18], differentiated TUS populations by seven common occupancy patterns (e.g. home all day, out from 9 am-6pm, etc.) across all household types. This method is suitable for generating household-type specific single-day profiles based on the proportion of each particular day type per defined population.

Analysis of the UK TUS data conducted by the authors determined that age, gender, and diary day employment status had a significant influence on occupancy characteristics, confirming consistency with the French TUS analysis performed by Wilke
[18].

To illustrate the relative influence of these additional factors, 1-person non-retired households were analysed; this group was selected as it allowed the influence of age, gender and employment status to be analysed in isolation from the influence of other occupants. Weekday, Saturday and Sunday datasets were considered separately allowing the effect of day type on occupancy to be assessed.

The overall 1-person non-retired household population was split based on working/non-working days, over-44/under-44 years old (selected based on a 50/50 dataset split and an observed occupancy behaviour change between 40 and 45) and the sex of the subject. The working characteristic is defined as those with more than 5 working hours during the diary day as this was seen to be the working duration at which this behaviour was distinguishable from typical non-work related daytime absences.

Table 1

Average weekday active occupancy probabilities for 1-person non-retired household sub-populations.

$\begin{array}{cc}\text { TUS Population } & \text { Average Active Occupancy Probability } \\ \text { Overall } & 0.317 \\ \text { Work-day / Non-Work-day } & 0.240 / 0.412 \\ \text { Under-44 / Over-44 } & 0.260 / 0.376 \\ \text { Male / Female } & 0.291 / 0.343\end{array}$

As shown in Table 1, diary day employment status had the greatest overall influence on average occupancy. Occupant age also was significant, with a consistent increase with age shown when multiple ranges are analysed.

Average active occupancy, is the average portion of the time during the day for the stated group when the occupant is awake and in the dwelling (i.e. sleep is excluded).

It should be noted that the current 24-hour basis for TUS diaries represents a fundamental barrier to considering any TUSbased occupancy models as being representative of individual households. A dataset of a similar scale, but with extended multi-day diaries, would be required to determine statistically how actual households compare to the average of their type.

Any differentiated model using current TUS data resolution would be indicative only of typical sub-population behaviour. Therefore, such models are most applicable for comparisons between sub-populations and for community-scale analysis, where a degree of averaging is acceptable.

\subsubsection{Duration Prediction}

According to Wilke [18], first-order Markov-Chain (MC) models result in overly random occupancy predictions with poor occupancy status duration prediction. Wilke proposed an alternative event-based model that uses the same TUS data to generate forward prediction of occupancy status and duration. This alternative method improved duration prediction, and reduced computational load with a recalculation per event rather than per time step. 


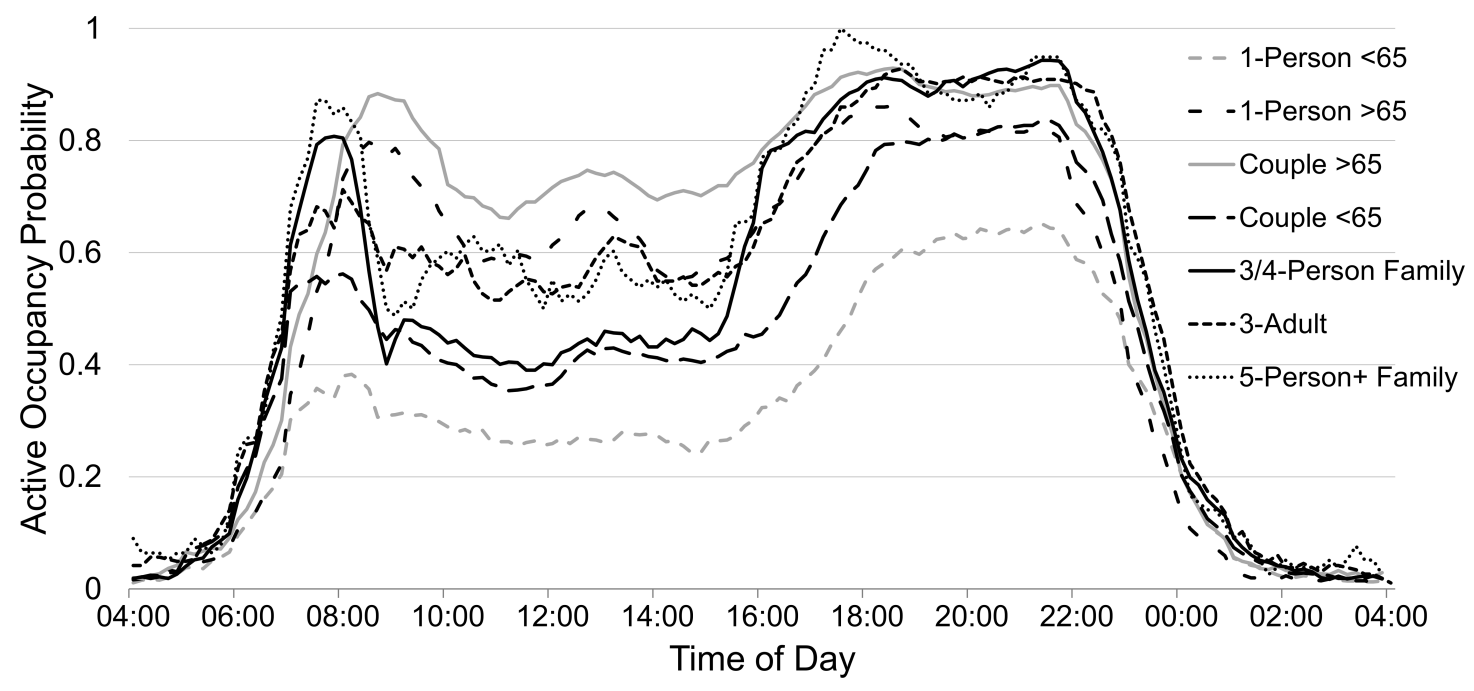

Fig. 2. Average weekday active occupancy probability profiles for different household types.

\subsubsection{Occupant Interactions}

For individuals in multi-person households, particularly those of co-habiting individuals (i.e. couples, parents), occupancy is not expected to be independent for each member.

Existing models either assume each household member is independent ([3], [17] and [18]) and accept the inherent error, or base the MC model on the number of active household members [13] but do not distinguish between individuals or differentiate by household type. The significance of this potential error has not been adequately determined.

\section{Aim and Contribution}

The aim and main contribution of this paper is to address the highlighted deficiencies in the prediction of occupancy using Markov-type models. To this end, a refined Markov-type model for occupancy prediction has been developed with the following innovations:

- First, transition probability matrices have been generated from the TUS dataset that differentiate between critical household characteristics with regards to the probability of active occupancy.

- Second, a higher-order Markov model was developed in order to improve the prediction of transitions and durations of different occupancy states.

- Finally, further refinements are made to the basic Markov model in order to account for the impact of relationships between co-habiting individuals on the overall active occupancy probability.

\section{An Improved Markov Model}

The basic large population, first-order Markov methodology, as used by [13] and others, has been adapted and refined in order to improve occupancy prediction. The nature of the changes are described below along with the verification of the benefits of these changes.

\subsection{Verification Mechanisms}

Three metrics are used in this paper to assess the impact of the changes made to the Markov-Chain occupancy model.

Average Occupancy Metric - determines the average pertime step occupancy error between the Time Use Survey (TUS) input data and model output for each occupancy state - quantifying the quality of calibration of the model. Equation (1) below is based on 144 data points per day (10 minute time steps).

$$
A O_{\text {state }}=\sum_{t=1}^{144} \frac{\left|\bar{P}_{\text {state }}^{\text {mod }}(t)-\bar{P}_{\text {state }}^{\text {tus }}(t)\right|}{144}
$$

where, $\mathrm{AO}_{\text {state }}$ is the Average Occupancy Metric for state, state, $\bar{P}_{\text {state }}^{\text {mod }}(\mathrm{t})$ is the average modelled probability for state, state, at timestep, $t$, and $\bar{P}_{\text {state }}^{\text {tus }}(\mathrm{t})$ is the average probability for state, state, at timestep, $t$, derived from the input Time-Use Survey data.

Two means of analysis are possible with this metric.

- First, it can be used to calculate the prediction for the average per time step results of multiple profiles generated using the model. This determines how effectively the model converges to the population average (hereafter referred to as AO_Conv).

- Second, it can be used to calculate the prediction error for each individual profile. The mean of this error can be used to determine how effectively individual profiles replicate the input data (hereafter referred to as AO_Var).

Over multiple profiles, a refined Markov model should be consistent with the input data. However, individual profiles should demonstrate deviation from the population average occupancy. Within real populations individual households will also deviate; so, a model that tracks the broad occupancy characteristics but with some variation about the mean is acceptable within limits.

State Duration Distribution Metric - (hereafter referred to as DurDist) is used to assess the ability of a model to generate a 
realistic range of occupancy state durations. It compares the difference in the cumulative probability function (CDF) at each 10-minute duration range for the histograms of the model generated results and TUS data in order to determine if the generated occupancy profile replicates the occupancy state durations seen in the TUS. The 'error' is the sum of the absolute difference between the model and TUS data CDFs at each duration value for each state.

This metric is commonly known as the Earth Movers Distance: a commonly used quantitative histogram similarity measure where the bin values are not independent and cross-bin analysis is required [14].

$$
\text { DurDist }_{\text {state }}=\sum_{d=1}^{144}\left|\sum_{d=1}^{d} \bar{P}_{\text {state }}^{\text {mod }}(d)-\sum_{d=1}^{d} \bar{P}_{\text {state }}^{\text {tus }}(d)\right|
$$

where, $\bar{P}_{\text {state }}^{\text {mod }}(d)$ is the probability of a modelled state duration of $d$ for state, state and $\bar{P}_{\text {state }}^{t u s}(d)$ is the probability of a state duration of $d$ for state, state, derived for the input Time-Use Survey data.

Occupancy Profile Similarity Metric - the process used is generally known as the Levenshtein Edit Distance Method (LEDM) for character string similarity analysis, which is used to compare individual occupancy profiles and is similar to the method used by [2]. The derived metric is hereafter referred to as ProfSim.

This LEDM method is used to quantify the dissimilarity between two strings by quantifying the measures needed to transform one into the other. In the LEDM a 'cost' of 1 is assigned for each edit (insertions, deletions, and replacements) required in the transformation. For example, transforming 110111 to 001011 would require a minimum edit of a replacement of the first digit, deletion of the second, and insertion of the last digit - a total cost of 3. The approach can therefore be applied when comparing two numerical profiles. When two profiles are compared, for clarity, the total 'cost' is converted from a per-time step to an hour equivalent by dividing the result by the number of time steps per hour.

The metric can be used in two ways.

- First, it can be used to compare the output profiles with the input dataset. The smallest cost per profile, representative of the closest match, is determined and an average calculated across all modelled days. This is a measure of the average similarity between generated profiles and the closest real profile.

- Second, each profile in either the input dataset or model output dataset can be compared with other profiles in the same dataset quantifying the behavioural similarity within and between each dataset.

There is no clear definition of when an input dataset, in terms of occupancy behaviour, is either overly similar or contains an unrepresentative population. Similarly there is no clear delineation of the point at which the output results change from overly random to realistic or from realistic to narrowly replicating the input data. The ProfSim metric does, however, allow a relative assessment to be made.
These comparison metrics will be used throughout the paper to gauge the relative effectiveness of each modelling method reviewed.

\subsection{Markov Model Improvements}

The following sections describe the development of a refined higher-order Markov-Chain model for occupancy prediction. Firstly, population-specific occupancy data is used to calibrate the model, secondly, the models prediction of occupancy state duration is improved, and finally, the influence of occupant relationships is accounted for. In this paper, the term 'higher-order' is used to define a model where existing state durations beyond the preceding time step are taken into account when determining the probability of a state transition.

\subsubsection{Occupant Differentiation}

The TUS dataset allows the number of occupants and their relationships to be easily extracted and characterised. In addition, data such as age, gender, employment hours, and diary date is included that potentially allows further relevant differentiation between occupant groups.

The TUS data analysis summarised in Table 1 showed that for 1-person households, employment status had the most significant impact on active occupancy, followed by age, and then gender. However, in developing a probabilistic, differentiated occupancy model, there is a need to balance the benefit of increased realism obtained by using smaller subgroups with a good depth of probability data from larger, heterogeneous populations.

To allow differentiation into sufficiently well-defined subpopulations and capture clear occupancy differences, whilst retaining a sufficient depth of data, the new model distinguishes between three basic occupancy states ('Sleep', 'Active' or 'Out') rather than multiple 'active' states as per the Widen et al [17], and Wilke [18] models. The need to distinguish between the 'Sleep' and 'Out' inactive states is necessitated by the differences in sleep and absence timings and durations that are utilised by the higher-order methods investigated later in the paper and also that whilst sleeping an individual will still contribute to heat gains in a dwelling. From this three-state basis, work was undertaken to quantify when a population was of the correct size to provide the ideal balance between characterising the behaviour of specific groups and being statistically robust.

A variety of methods were used to attempt to identify the minimum population size required to produce a robust statistical model. Edit distance (ProfSim) analysis indicated that the minimum number of TUS diaries required to produce a model that generated sufficient behavioural variety was approximately 100 .

Two further methods were used to determine the potential for producing robust models. One was to review the number of probability coefficients in the Transition Probability Matrices (TPMs) greater than zero and less than one. A zero value indicates that there was no individual with that specific state transition and a value of one is typically associated with the behaviour of one person (and is therefore not necessarily representative of wider behaviour). A fractional value requires mul- 
tiple people to be represented and the number of such elements can be used as a proxy for probability data quality, and it is assumed, by extension, model stability.

The other was to review the number of times an annual higher-order model had a state and duration range that did not have associated probability data and required a recovery function to be used. This can occur due to the use of duration ranges rather than specific durations in the model calibration resulting in scenarios not seen in the input data. This is also an indirect measure of data quality as reducing the use of the recovery function requires an increasing likelihood of non-zero probability data for transitions in adjacent duration ranges.

Example results for both measures for the under 65, nonworking population are shown in Table 2.

\section{Table 2}

Fractional TPM elements and recovery function use for different sizes of transition probability input datasets (under-65, non-working, 1-person households).

\begin{tabular}{ccc}
\hline Households in Dataset & $\begin{array}{c}\text { Fractional TPM } \\
\text { Probability Elements } \\
(\text { out of 9072)* }\end{array}$ & $\begin{array}{c}\text { Time-steps Recovery } \\
\text { Function Required } \\
\left(\times 10^{-3} \%\right)\end{array}$ \\
\hline 50 & 531 & 1.81 \\
100 & 834 & 0.93 \\
150 & 1084 & 0.64 \\
200 & 1394 & 0.42 \\
400 & 1891 & 0.31
\end{tabular}

* There are a large number of unlikely transitions which is why the number is low compared to total elements.

Both measures improve exponentially with an increasing number of input diaries, with the most significant improvement up to c. 200 diaries. This suggests that 200 diaries should be the target for stable statistical modelling. It was therefore decided to split the populations by appropriate age range but not gender at this stage to maintain sufficiently sized populations for groups that remain small enough to capture differentiated behaviours. Gender being the least significant of the four key differentiating elements identified (household type, employment status on model day, age and gender). As an example, the single household population shows distinct behaviour changes around 40-45 years of age, at retirement age, and around 70-74 years of age that are consistent for both genders.

To maximise the benefit of differentiation by age, the TUS dataset was split into overlapping age ranges. For working oneperson households, the 18-37 TUS population was used for the 18-33 model data, the 28-44 population for the 34-40 model, etc. This increases the number of diaries per population group and also recognises that the age-related behaviour changes are gradual and that single day diaries may not adequately capture more extreme behaviours within groups. Splitting the population to account for the identified age-related behaviour transitions results in groups of approximately 200-250 diaries, with an approximate 50/50 gender split.

\subsubsection{Differentiated Model Verification}

To analyse the impact of using smaller, differentiated individual occupant populations, a general 1-person household occupant model (representative of the models developed by [13] and [17]) is compared to a model calibrated using two sub- populations from the TUS dataset: 'Working 18-37' - working individuals between 18 and 37 years of age, and 'Over 76' - typically retired individuals over 76 years of age. A firstorder Markov model method was used for a typical weekday to predict occupancy state ('Sleep', 'Active' or 'Out'), with 100 1-year duration 10-minute timestep occupancy state sequences generated for each case.

The results were analysed using the average active occupancy variation metric (AO_Var) to determine the degree of calibration (see Table 3): comparing the mean error between the predicted active occupancy per modelled annual sequence and that found in the input dataset.

In Table 3, 'All 1-person' represents the model calibrated with the entire TUS 1-person dataset, 'Working 18-37' and 'Over 76' represent the models calibrated using the identified TUS subgroups.

Table 3

Per-profile average occupancy probability variation analysis for different firstorder model populations.

\begin{tabular}{ccc}
\hline Model & TUS Population & AO_Var \\
\hline All 1-person & All 1-person & 0.020 \\
All 1-person & 'Working 18-37' & 0.225 \\
All 1-person & 'Over 76' & 0.139 \\
'Working 18-37' & 'Working 18-37' & 0.014 \\
'Over 76' & 'Over 76' & 0.017
\end{tabular}

The results indicate that the first-order Markov model, calibrated using the more refined datasets produces occupancy behaviour that is more representative of those subgroupings within a population, as opposed to the model calibrated using the larger dataset.

The state duration prediction comparison between the models calibrated using the same populations is shown in Table 4. (The values quantify the difference (measured using metric DurDist) between the actual occupancy duration distribution and the distribution predicted using the model.)

Table 4

State duration analysis (DurDist) for different first-order model populations.

\begin{tabular}{ccccc}
\hline Model & TUS & Sleep & Active & Out \\
\hline All 1-person & All 1-person & 1.73 & 1.13 & 4.04 \\
All 1-person & 'Working 18-37' & 8.20 & 9.72 & 22.43 \\
'Working 18-37' & 'Working 18-37' & 2.26 & 0.79 & 2.87 \\
All 1-person & 'Over 76' & 4.13 & 7.11 & 8.93 \\
'Over 76' & 'Over 76' & 1.64 & 1.63 & 1.10
\end{tabular}

Significant improvements are again shown where both the model input data and comparison TUS data are from the same population. There is, however, a further improvement in the DurDist metric for the smaller populations. More importantly, the results show that the overall 1-person household population significant fails to properly replicate the range of durations for the two identified sub-populations.

The ProfSim metric is used to identify the lowest edit distance for each day in the output from the differentiated and larger population models in comparison with the input data. This allows an assessment of the models ability to generate realistic profiles. In this example, the overall 1-person house- 
hold ('All 1P') first-order model was again compared with the 'Working 18-37' and 'Over 76' sub-population models. The average minimum edit distance (expressed as a time) for the 'All 1P' model compared to the 'Working 18-37' TUS dataset is 4.35 hours. The result when the 'Working 18-37' specific model is used is 1.75 hours. The equivalent improvement for the 'Over 76' population was from 2.71 to 1.98 hours. This suggests that the expected improvement from differentiation reduces for sub-populations with less consistent behaviour patterns.

Overall, there is an improvement in the first-order Markov models ability to replicate observed behaviour using smaller sub-populations. The degree of improvement would depend on the deviation of the sub-population from the overall population average, but is significant for those with extreme deviations.

\subsubsection{Occupancy Status Duration}

As was mentioned previously, first-order Markov occupancy models do not consider the duration of the current state and are therefore 'memoryless'. The state at the next time step being dependent only on the previous time step state. It was determined by Wilke [18] that such models are likely to poorly predict state durations. This is demonstrated using a three-state ('Sleep'/'Active'/'Out') first-order Markov model for 100 1person households over an annual (52560 time step) run. The predicted cumulative probability for the 'Out' duration is shown in Figure 4 along with the actual cumulative duration of this state from the TUS dataset. The results show that the first-order model averages duration probability and does not capture the tendency for absences to be either short or long. This discrepancy is more significant for working days.

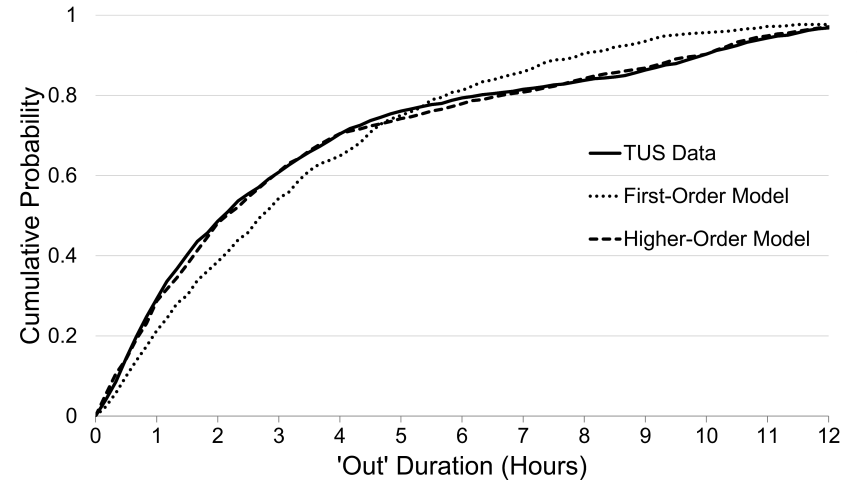

Fig. 4. Cumulative distribution of Out state duration for all 1-person households.

Similar results occur for the sleep and active states. The firstorder model therefore generates occupancy patterns which often do not reflect reality and the conclusion is that it cannot accurately capture specific behaviours (e.g. sleep, work absences etc.).

To improve the prediction of state duration, a simple higherorder Markov method was developed where multiple probability transition matrices have been generated according to the duration of the existing state, accounting for behaviour prior to the previous time step. A single transition probability matrix is therefore replaced with matrices corresponding to, for example, sleep durations of 0-2, 2-4, 4-6, 6-8, and 8+ hours. So, if an occupant has been asleep for 3 hours then the 2-4 hour sleep duration transition probability matrix would be used to determine the next occupancy state. This approach captures the changes in relative probability of waking having slept for different lengths of time. Optimum ranges vary per transition based on specific behaviours, and in particular, those related to sleep and work-related absences.

The difference in the per-time-step transition matrices for the first and higher-order models is shown in Figure 3. Each row of the first-order matrix transforms into a multi-row matrix. ('P' is the probability of a particular transition, ' $S$ ' refers to 'Sleep', ' $\mathrm{A}$ ' is 'Active' and ' $\mathrm{O}$ ' is 'Out'). For example, the matrix element on the third row and second column represents the probability that someone who has been asleep for between 4 and 6 hours at a particular time step will transition to the 'Active' state.

\subsubsection{Higher Order Model Verification}

In order to assess the relative performance, the new higherorder model is first compared to the original equivalent firstorder Markov model, and then compared to a higher-order event-based model (see Validation section below). As before, the analysis is based on results for 100 1-person households over an annual (52560 time step) run.

Results for the average active occupancy prediction metrics (AO_Conv) and (AO_Var) showed no significant performance difference between both Markov-based methods, suggesting both capture basic occupancy probability to the same degree.

Using metric 'DurDist', the distribution of state durations was compared for the first-and higher-order models for two different sub-populations; the 18-37 working population and the over 76 non-working population (see Table 5).

Table 5

State duration analysis (DurDist) for first and higher-order Markov models.

\begin{tabular}{cccc}
\hline Model & Sleep & Active & Out \\
\hline 'Working 18-37' First-Order & 2.26 & 0.79 & 2.87 \\
'Working 18-37' Higher-Order & 1.42 & 0.47 & 1.43 \\
'Over 76' First-Order & 1.64 & 1.63 & 1.10 \\
'Over 76' Higher-Order & 1.26 & 1.15 & 1.03
\end{tabular}

The results shows that the higher order model gives improved prediction of state durations when compared to the input TUS data, particularly for the working population. Similar results were achieved for other populations. This improvement is also demonstrated graphically in Figure 4 for the overall 1-person population model, with the higher-order model results tracking the input data distribution more closely.

Table 6 shows the same profiles assessed for similarity using the edit distance method (ProfSim), this again shows a demonstrable improvement when using the higher-order model in the ability to generate realistic profiles. This improvement was more significant in sub-populations of the TUS dataset with more distinct behaviours, confirming the benefit of the higherorder approach is dependent on the sub-population behaviour consistency. 


\begin{tabular}{|c|c|c|c|c|c|c|c|}
\hline & \multirow[b]{3}{*}{ Sleep } & \multirow[b]{3}{*}{ Active } & \multirow[b]{3}{*}{ Out } & $\begin{array}{l}\text { Current } \\
\text { State / Duration }\end{array}$ & Sleep & Active & Out \\
\hline & & & & Sleep / 0-2 hrs & $P_{S 2} \rightarrow s$ & $P_{S 2} \rightarrow A$ & $P_{S 2} \rightarrow 0$ \\
\hline & & & & Sleep / 2-4 hrs & $P_{S 4} \rightarrow S$ & $P_{S 4} \rightarrow A$ & $P_{S 4} \rightarrow 0$ \\
\hline \multirow[t]{3}{*}{ Sleep } & $P_{S} \rightarrow s$ & $P_{S} \rightarrow A$ & $P_{S} \rightarrow 0$ & Sleep / 4-6 hrs & $P_{S 6} \rightarrow s$ & $P_{S 6} \rightarrow A$ & $P_{S 6} \rightarrow 0$ \\
\hline & \multirow{2}{*}{\multicolumn{3}{|c|}{ First-Order }} & Sleep / 6-8 hrs & $P_{S 8} \rightarrow S$ & $P_{S 8} \rightarrow A$ & $P_{S 8} \rightarrow 0$ \\
\hline & & & & Sleep / 8hrs+ & $P_{S 8+} \rightarrow S$ & $P_{S 8+} \rightarrow A$ & $P_{S 8+} \rightarrow 0$ \\
\hline
\end{tabular}

Higher-Order

Fig. 3. Transition from a first-order to a higher-order Markov model (Sleep state example).

Table 6

Occupancy profile similarity analysis (ProfSim) for first and higher-order oneperson household models.

\begin{tabular}{cc}
\hline Model & LEDM (Hours) \\
\hline 'Working 18-37' First-Order & 1.75 \\
'Working 18-37' Higher-Order & 1.53 \\
'Over 76' First-Order & 1.98 \\
'Over 76' Higher-Order & 1.92
\end{tabular}

The edit distance method can also be used to assess datasets for similarity. Each single-day occupant profile (input data and output model) are compared with the other profiles in the same dataset and the edit distances determined. The distribution of edit distances (see Figure 5) demonstrates graphically the overall similarity between profiles in a particular dataset.

Figure 5 shows the results from the TUS dataset and different model types for the 'Working 18-37' population. The horizontal axes elements represents the edit distance rounded up to the nearest hour and the vertical axis is the proportion of all edit distances within each edit distance range. (For example, horizontal axes element ' 9 ' represents the edit distances in the range $8<\mathrm{ED} \leq 9$ hours, for which the proportion was 0.073 in the TUS 2000 dataset.)

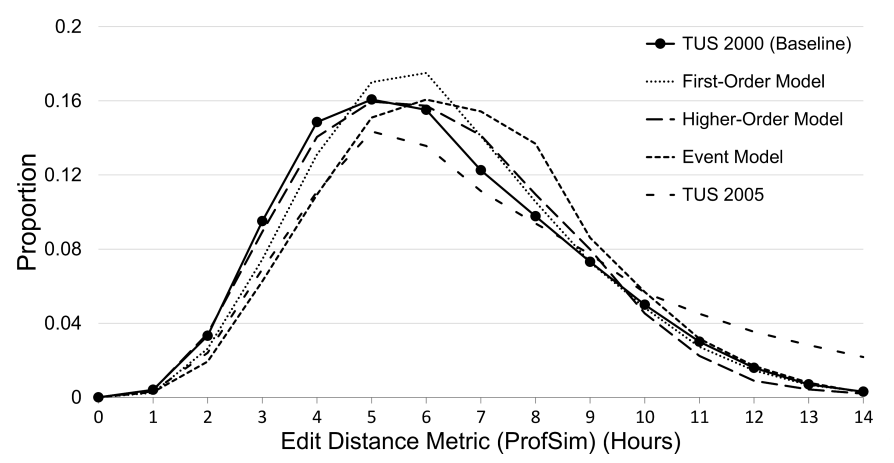

Fig. 5. Edit distance distributions for input and output datasets ('Working 1837 ' population).

An effective output model should replicate the input data baseline distribution. How far the peak value (and, by extension, the mean) of the distribution has increased is a measure of the overall increase in dissimilarity. A narrower overall distribution suggests a model that produces less realistic averaged profile outputs.

Of the three modelling methods, the higher-order model pro- duces the closest approximation of the input dataset similarity distribution. The distribution shows a slight decrease in overall similarity and a reduction in the number of highly dissimilar profiles produced, but is closer than the other two methods. In comparison, the first order model shows a distinctly narrower overall distribution, suggesting a tendency to replicate average rather than generate realistic individual behaviours. This confirms the results shown in Tables 5 and 6 which demonstrate that the higher-order model better replicates both state durations (metric DurDist) and actual profiles in the input dataset (metric ProfSim).

\subsubsection{Interaction of Couples, Parents and Children}

In existing models, co-habiting couples were either modelled as independent adults ([17], [18]) or not distinguished from other households with the same occupant number [13]. However, analysis indicates that this leads to discrepancies in the estimation of the number of occupants per-time step and an overestimation of total occupied time (see Figure 6).

To improve interaction prediction, each couple was instead modelled as a single entity, having a status based on both individual states. To minimise the data requirement, and assuming tracking specific individuals is not critical, the individual states are unassigned (e.g. Sleep/Active combines Sleep/Active and Active/Sleep etc.).

The average age of the couple is used to define age ranges as this was shown by analysis of the TUS dataset by the author to be a better differentiator for occupancy than individual ages. Days with both occupants working and one occupant working were also differentiated in the generation of the transition probability matrices.

Occupancy states from the combined couple model was compared to the predictions based on two adult individuals. The joint model results are significantly closer to the input TUS dataset. As an example, Figure 6 shows the results for the working age 28-50 sub-population.

In Figure 6, 'Any Active' represents the probability of either 1 or 2 people being active and in the dwelling. The 'TUS' elements are the baseline results from the input TUS dataset. The 'Individual' elements are the results for the multiple individual models method, and 'Combined' the results for the single combined model method. Similar results can be shown for the prediction of periods where 1 person is active and in the dwelling.

This combined method was also applied separately to parents with resident children. Parent occupancy patterns were 


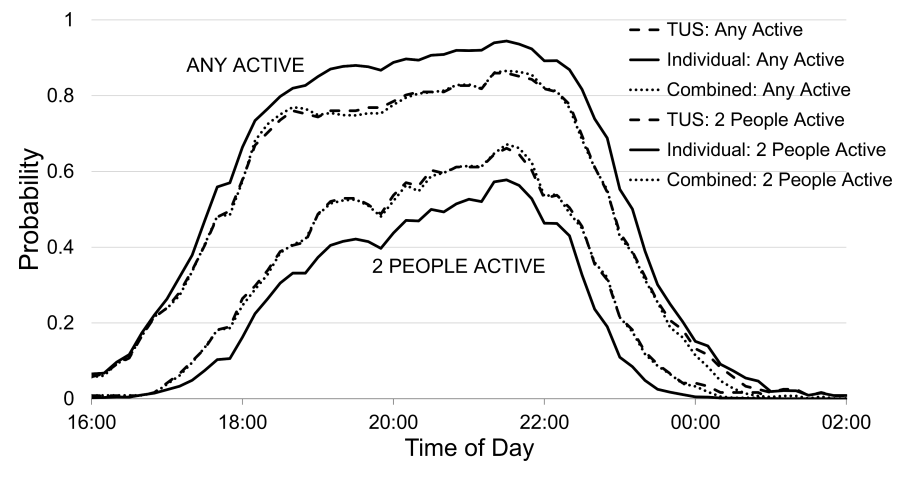

Fig. 6. Impact of Combined Couple Model on Individual and Overall Occupancy.

assumed to be at least partially driven by child occupancy requirements, and that the child model could therefore be simplified and linked directly to the parent model. The child model is also Markov-chain based, but is first-order and only tracks whether the child is active or inactive. For a child, 'sleep' and 'out' distinctions for the 'inactive' state can be inferred based on time-of-day.

The child model utilises transition probability matrices (TPMs), determined from the TUS dataset, that reflect the probability of a change in child occupancy state dependant on a particular transition in parent state. The parent model is therefore run first to determine the parent state at the new time step. For example, at time step, $t-\Delta t$, parent occupancy is one active/one inactive and the child is inactive and, at timestep, $t$, parent occupancy becomes both active. The selected TPM for the child model is the one that determines whether the child remains inactive or becomes active if a second parent becomes active. Similar TPMs are available for all potential parent occupancy transitions (including no change), for both potential initial child states.

Whilst the couple model uses average age for differentiation, it was determined that the age of the youngest child was the strongest determinant of parent occupancy. This was done by comparing the relative influence of a variety of factors (i.e. average parent age, age of oldest parent, age of youngest parent, average child age, age of eldest child) on overall occupancy probability and selecting the one which showed the most distinct occupancy variations between sufficiently-sized populations for effective modelling.

Each child was modelled separately as there is insufficient data to determine occupancy interaction between siblings of different ages. Child occupancy was split by age range (e.g. 8-9, 10-11 etc.), and between school-term and holiday periods.

Separate independent models are used for 'adult' children living in the parental home. One for 16-18 year olds in education, and the other for the remainder of 16-24 year olds. It can be shown that their occupancy is broadly independent of the other household members.

\subsubsection{Couple Model Verification}

Analysis with the average occupancy prediction (AO) metrics is less straightforward for 2-person models as either simple ac- tive occupancy (Any Occ) or the specific occupant number (Occ Num) can be analysed. Table 7 shows the results for the average active occupancy variation metric (AO_Var) analysis, considering both options, for working couples with an average age between 28 and 50 ('Working 28-50' model population). For the specific occupant number the total error is the sum of the errors for single and double occupancy prediction compared to the input TUS dataset.

Table 7

Average occupancy prediction comparison between combined and multiple individual model options ('Working 28-50' population).

\begin{tabular}{ccc}
\hline Model & $\begin{array}{c}\text { AO_Var } \\
\text { (Any Occ) }\end{array}$ & $\begin{array}{c}\text { AO_Var } \\
\text { (Occ Num) }\end{array}$ \\
\hline 2 x Individual First-Order & 7.17 & 15.70 \\
2 x Individual Higher-Order & 6.40 & 14.56 \\
'Combined' First-Order & 5.05 & 6.70 \\
'Combined' Higher-Order & 3.71 & 5.04
\end{tabular}

The results demonstrate both the improvement switching from independent to combined models, and also the additional improvement of the higher-order model in comparison to the equivalent first-order model when applied to the combined couple model.

The status duration comparison metric (DurDist) for the 'Working Couple 28-50' model (see Table 8) shows a significant improvement using the combined model approach, and a more limited additional benefit from using the higher-order Markov approach for this particular metric.

Table 8

State duration analysis (DurDist) for first and higher-order Markov 'Working Couple 28-50' models.

\begin{tabular}{ccccccc}
\hline Model & S-S & S-A & S-O & A-A & A-O & O-O \\
\hline 2 x Individual First-Order & 3.53 & 1.33 & 0.85 & 1.54 & 1.44 & 2.97 \\
2 x Individual Higher-Order & 2.59 & 1.45 & 0.75 & 1.84 & 0.88 & 2.12 \\
'Combined' First-Order & 0.99 & 0.37 & 0.84 & 0.65 & 0.30 & 1.67 \\
'Combined' Higher-Order & 0.97 & 0.29 & 0.88 & 0.50 & 0.30 & 1.36 \\
'S'=Sleep, 'A'=Active, 'O'=Out & & & & &
\end{tabular}

In Table 9 results for occupancy profile analysis (metric ProfSim of the same population shows a more significant benefit for the higher-order approach.

Table 9

Occupancy profile analysis (ProfSim) for first and higher-order Working Couple 28-50 models.

\begin{tabular}{cc}
\hline Model & LEDM (Hours) \\
\hline 2 x Individual First-Order & 3.88 \\
2 x Individual Higher-Order & 3.38 \\
'Combined' First-Order & 3.28 \\
'Combined' Higher-Order & 2.89
\end{tabular}

Considering all results, quantitative and graphical, the singleentity, higher-order model provides an improved method for predicting the occupancy for related, co-habiting households.

\subsubsection{Family Model Results}

As outlined above, the two-parent family model combines the method for co-habiting couples with a simple child model linking child occupancy directly with parent occupancy. 
The combined higher-order parent model exhibits similar metric improvement as for couple models. Figure 7 shows that the model tracks the average total occupancy of the parents in a one-child household with good accuracy. It also demonstrates that the child model tracks the input data reasonably well with some short periods of relatively weaker agreement (late afternoon, mid-evening).

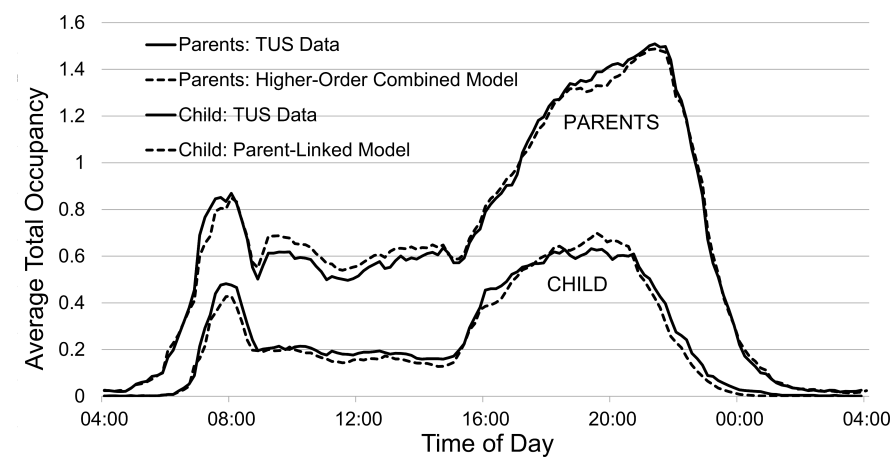

Fig. 7. Comparison of Average Child Active Occupancy (1-Child Households).

\section{Validation}

\subsection{Comparison with Alternative Approaches}

The previous analysis demonstrates that the higher-order Markov-Chain approach performs better than a first-order approach in terms of prediction of duration of occupancy events. Wilke [18] demonstrated that an alternative higher-order approach which seeks to identify each transition 'event' and subsequent duration probabilistically also shows an improvement over the first-order Markov approach, particularly for differentiated populations. It is therefore useful to compare the higherorder Markov and event-based occupancy prediction methods.

The 'event' model used in this comparison is similar to that developed by Wilke [18] (ibid) but uses the same three-state basis ('Sleep'/'Active'/'Out') as the Markov model. Wilke's original model predicted whether an individual was undertaking specific activities, as recorded in the TUS dataset.

For this paper, the Time Use Survey (TUS) dataset was used to derive probabilities for each potential occupancy state transition and the duration of each occupancy state. For each 10minute TUS data time step and for each of the three possible states the model calculates:

- if there is a change of state;

- the new transition state probability;

- the probabilities of the new state duration being within a particular range.

For example, Figure 9 shows the transition and duration matrices for an 'active' period that ends at $11.10 \mathrm{pm}$. The 'event' model will first generate a random number (RN1) between 0 and 1 to determine if the transition is to 'Sleep' or 'Out' states. The duration in hours of the new state is determined in the same manner using the duration probability matrix for the new state if starting at $11.10 \mathrm{pm}$. A third random number (RN3) determines with equal probability the exact 10-minute timestep on which the next transition occurs and the process is repeated for this identified next event. Using this approach, the model calculates a sequence of occupancy states and their durations, calculated to a 10 -minute resolution.

\begin{tabular}{|c|c|c|c|}
\hline \multirow{3}{*}{$\begin{array}{c}\text { Sleep } \\
\text { Duration } \\
\text { (Hours) }\end{array}$} & \multirow{3}{*}{$\begin{array}{l}\text { Cumulative } \\
\text { Probability } \\
\text { at } 11.10 \mathrm{pm}\end{array}$} & $\begin{array}{l}\text { 'Active' Period } \\
\text { ends at 11.10pm }\end{array}$ & $\begin{array}{l}\text { New State } \\
\text { Probability }\end{array}$ \\
\hline & & Sleep & 0.811 \\
\hline & & Out & 0.189 \\
\hline $0-1$ & 0.031 & \multirow{2}{*}{\multicolumn{2}{|c|}{$\begin{array}{c}\downarrow \\
\text { Random Number } 1(\mathrm{RN} 1)=0.336 \\
\rightarrow \text { New State }=\text { Sleep }\end{array}$}} \\
\hline $1-2$ & 0.078 & & \\
\hline $2-3$ & 0.093 & \multicolumn{2}{|c|}{$\rightarrow \rightarrow$ inew } \\
\hline $6-7$ & 0.449 & \multirow{2}{*}{\multicolumn{2}{|c|}{$\begin{array}{l}\text { Random Number } 2(\mathrm{RN} 2)=0.583 \\
\rightarrow \text { Sleep duration }=7-8 \text { hours }\end{array}$}} \\
\hline $7-8$ & 0.640 & & \\
\hline 8-9 & 0.813 & \multirow{2}{*}{\multicolumn{2}{|c|}{$\begin{array}{l}\text { Random Number } 3(\mathrm{RN} 3)=0.178 \rightarrow \\
\text { sub-hour time step }=2^{\text {nd }}=10-19 \mathrm{mins}\end{array}$}} \\
\hline $22-23$ & 1.000 & & \\
\hline \multirow[t]{4}{*}{$23-24$} & 1.000 & & \\
\hline & & \multicolumn{2}{|c|}{$\begin{array}{l}\text { Total Duration of Sleep State } \\
=7 \mathrm{hrs} 10-19 \mathrm{mins}\end{array}$} \\
\hline & & & \\
\hline & & \multicolumn{2}{|c|}{$\begin{array}{l}\text { Time step of next state transition } \\
\text { 'event' }=6.20-6.29 \mathrm{am}\end{array}$} \\
\hline
\end{tabular}

\begin{tabular}{|c|c|}
\hline $\begin{array}{c}\text { Out } \\
\text { Duration } \\
\text { (Hours) }\end{array}$ & $\begin{array}{c}\text { Cumulative } \\
\text { Probability } \\
\text { at 11.10pm }\end{array}$ \\
\hline $0-1$ & 0.286 \\
\hline $1-2$ & 0.345 \\
\hline $2-3$ & 0.360 \\
\hline $6-7$ & 0.812 \\
\hline $7-8$ & 0.888 \\
\hline $8-9$ & 0.939 \\
\hline $22-23$ & 1.000 \\
\hline $23-24$ & 1.000 \\
\hline
\end{tabular}

Fig. 9. 'Event' model next state type and duration calculation example.

The performance of the two higher-order methods was compared. The three metrics defined previously were again used to assess performance. Four single household TUS weekday populations were analysed: 'Working All Ages', 'Working 18-37', 'Retired All Ages' and 'Over 76'.

The average results for 1000 annual runs were compiled and are shown in Table 10. All results show a significantly better performance for the Markov model, particularly an order of magnitude improvement in overall convergence to the input data (AO_Conv) and significant improvement in the duration prediction metric (DurDist).

The occupancy profile similarity metric (ProfSim) was used for single-day profile analysis as before. For the 'Working 1837 ' population the average edit distance for the 'event' model was 1.98 hours, which compares poorly with 1.53 and 1.75 hours for the higher-order and first-order Markov methods respectively.

The occupancy profile similarity analysis (ProfSim) shown in Figure 6, shows that the profile similarity to the input TUS dataset is lower for the 'event' model in comparison to the higher-order Markov method. This can be inferred from the greater rightward shift from the TUS-derived distribution for the 'event' model, highlighting higher overall ProfSim values (and lower similarity). This shows that the 'event' method shortcomings addressed in this paper result in poor basic performance to replicate actual profiles.

Figure 8 shows the average per-time step active occupancy (AO_Var) error calculated from 1000 runs for the 'event', higher-order Markov and first-order Markov models. For the 'Working 18-37' 1-person population, the majority of the error for all models is during the morning and early evening periods. Both times correspond to significant changes in occupancy probability, which the 'event' approach fails to capture as effectively as both Markov methods as demonstrated by more significant peaks.

As state transitions have a low occurrence in the dataset, for 
Table 10

Metric comparison for developed higher-order models.

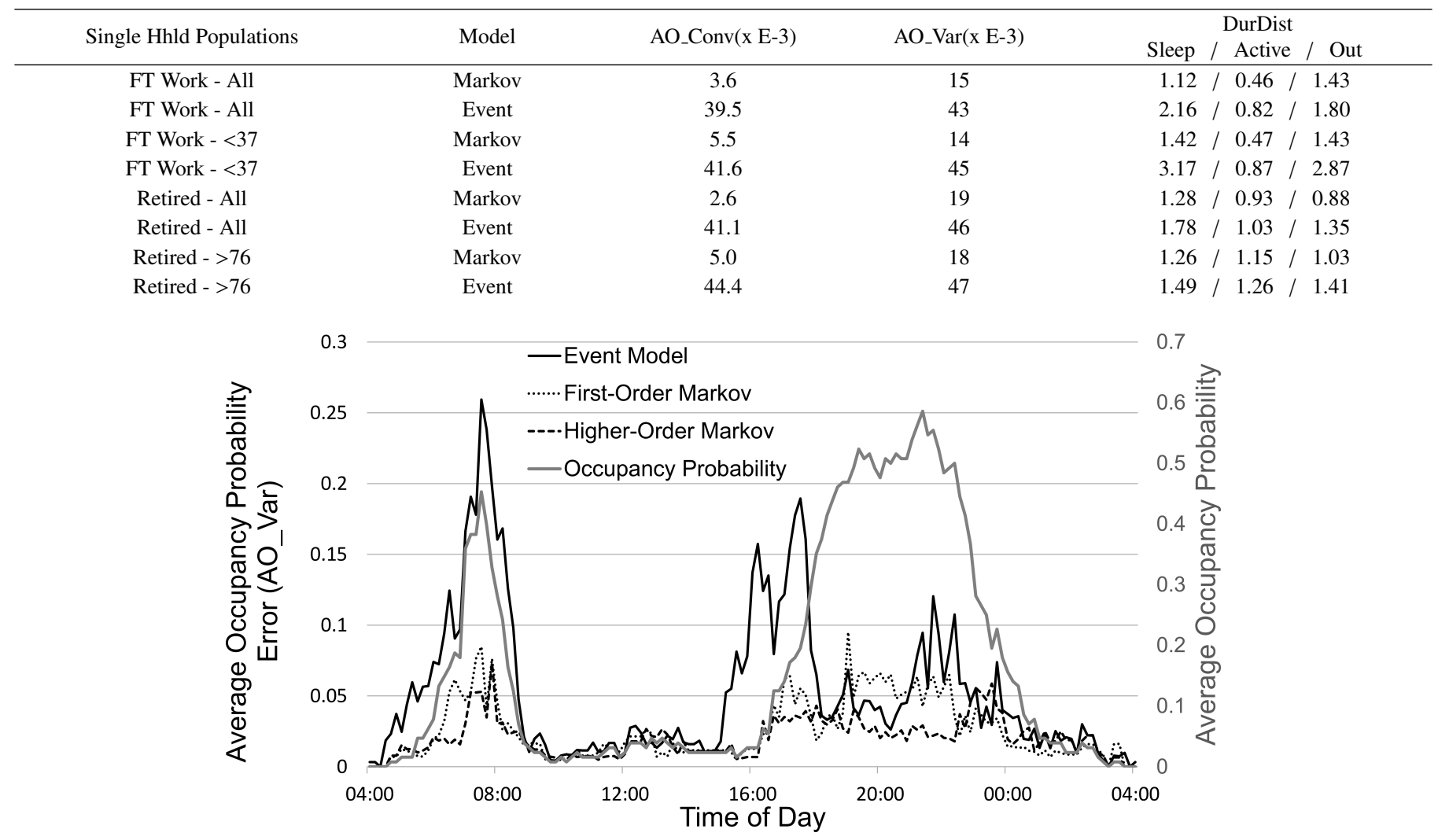

Fig. 8. AO_Var error time distribution comparison for each method.

each time step in the 'event' model, data from several adjacent time steps is used to ensure a sufficient depth of data. As with the Markov model, duration ranges are required rather than capturing specific durations. The base analysis presented above used three preceding and subsequent time steps for probability data, and 1-hour duration ranges.

Comparative analysis was undertaken with the event-based model to determine if the weaker performance was a result of the number of adjacent time steps or the duration ranges selected. Analysis with five and seven adjacent time steps, and 20 and 30-minute duration ranges, showed no significant change, therefore the errors seem to be inherent to the basic method.

One possible explanation for these results is that the eventbased method does not have the self-correcting nature of a pertimestep probability model. The balance of this method is too focused on state duration prediction at the expense of state probability based on time-of-day. Further, not effectively tracking basic daily behaviour also compromises the duration prediction as demonstrated by poor duration (DurDist) and occupancy profile similarity (ProfSim) metric results. This is illustrated by a detailed review of model outputs, which show an increased tendency for the 'event' model to produce unusual behaviours (e.g. no daily sleep period, less distinct work-related absences, etc.).

\subsection{Independent Dataset Validation}

For final validation of the differentiated, higher-order model, the results were compared with occupancy profiles from the smaller 2005 UK TUS survey [12]. This dataset uses a simpler UK-specific methodology with 4941 diaries compared to 20981 for the 2000 TUS survey [11].

For validation purposes, both TUS datasets should capture similar occupancy behaviour. Figure 10 demonstrates that the average weekday profile for the overall 1-person household population and the two 1-person sub-populations (under 37 years old on working days and over 80 years old) analysed in detail for this paper are broadly consistent. This confirms that there are occupancy traits that are inherent to the TUS subpopulations, which was also confirmed for other occupant and household types.

In order to discount the differences between the TUS datasets in the comparison, the two TUS datasets were first compared using the same metrics deployed in the comparison between the two Markov models and the TUS data. If the models are successfully predicting occupant behaviour then there should not be a significant increase in the model-TUS comparison metrics compared to the inter-TUS metrics.

The results in Table 11 show analysis of the average active occupancy (AO), duration prediction (DurDist), and profile similarity (ProfSim) metrics. The results for both the first and higher-order models compared to the TUS 2005 dataset are 
Table 11

Metric results for TUS 2000 data and Markov models compared to TUS 2005 data.

\begin{tabular}{|c|c|c|c|c|}
\hline Dataset 1 & Dataset 2 & AO_Var & ProfSim (Hours) & $\begin{array}{ll} & \text { DurDist } \\
\text { Sleep / Active / Out }\end{array}$ \\
\hline 'Working 18-37' TUS 2000 & 'Working 18-37' TUS 2005 & 4.69 & 11.9 & $2.55 / 1.27 / 4.20$ \\
\hline 'Working 18-37' First-Order & 'Working 18-37' TUS 2005 & 4.75 & 12.1 & $2.67 / 1.23 / 3.25$ \\
\hline 'Working 18-37' Higher-Order & 'Working 18-37' TUS 2005 & 5.15 & 11.7 & $2.17 / 1.23 / 4.19$ \\
\hline 'Over 76' TUS 2000 & 'Over 76' TUS 2005 & 4.15 & 12.4 & $4.50 / 3.44 / 3.76$ \\
\hline 'Over 76' First-Order & 'Over 76' TUS 2005 & 5.21 & 12.3 & 4.40 / 3.35 / 3.49 \\
\hline 'Over 76' Higher-Order & 'Over 76' TUS 2005 & 4.91 & 12.3 & $4.72 / 3.58$ / 3.83 \\
\hline
\end{tabular}

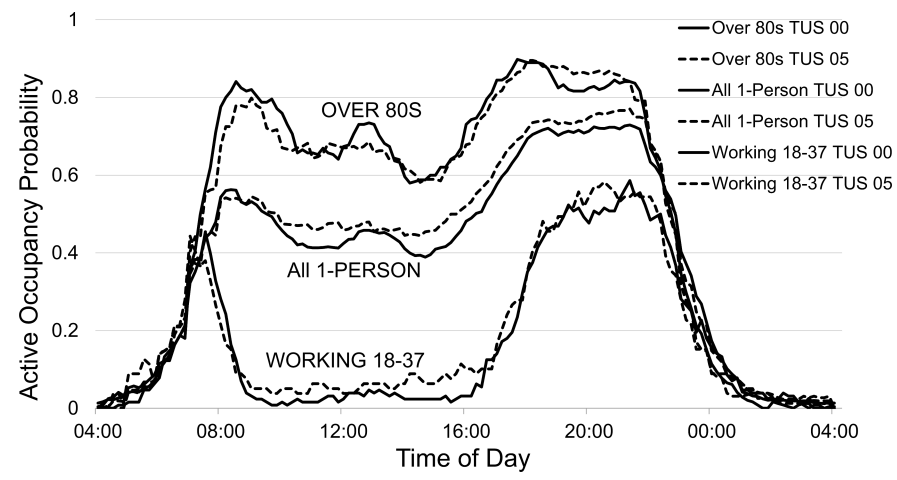

Fig. 10. Single household average active occupancy profiles comparison for TUS 2000 and 2005 datasets.

broadly consistent with the calculated difference between the two TUS datasets. This demonstrates that the sub-population behaviours are adequately replicated, despite some differences between the two TUS populations.

The results are less conclusive regarding the performance of the higher-order model relative to the first-order model when compared with the 2005 dataset. Both methods perform slightly better on some measures, and worse on others. The TUS 2000 dataset may be too small to produce wholly representative data for the sub-populations. Alternatively, there may be an inherent weakness in the metrics used to differentiate relative performance at this level of similarity. Further analysis with the larger 2015 TUS dataset will be required for a better judgement of the higher-order model benefit relative to normal variability rather than merely to the input data.

\section{Final Differentiated Model Basis}

\subsection{Occupancy Model Selection}

All defined metrics show a clear advantage for the higher-order Markov method over the 'event' method. There was no obvious difference in relative performance based on type or size of population selected.

The benefits of the higher-order Markov method compared to the first-order method are less conclusive. There is a measureable improvement in the metrics for duration and consistency with actual TUS profiles, especially for groups with consistent patterns of behaviour (e.g. workers). In comparison with the independent TUS dataset the results are less clear. However, there is sufficient justification to use the higher-order method for further development as there is evidence that the residual is- sues are related to current data availability rather than the basic method.

The improvements in all defined metrics for the differentiated model suggests that the level of differentiation used with a minimum of 200 diaries per sub-population was sufficient to produce stable models that are at least representative of the input data.

\subsection{Model Structure}

\subsubsection{Occupant Type Modules}

The developed model integrates the three basic modules; single-person, couple, and child, outlined in the preceding sections, with further differentiation by age, employment status and day type as proposed.

The single-person model can be used for single households, and for individuals in multiple unrelated adult households or households with related adults of different generations (e.g. adult children) with divergent behaviours. It has seven age ranges from 18-33 to 80+ and two further models for young adults living in a family household; 16-18 year olds in education and a general 16-24 age group.

The couple model has separate probability data for cohabiting couples with and without dependent children. The 'without children' dataset has seven age ranges based on average age. The 'with children' dataset has 4 ranges based on the youngest child's age.

The child model has 5 age ranges $(5-7,8-9,10-11,12-13$, and 14-15). Under $5 \mathrm{~s}$ are not modelled due to the lack of TUS data for infants, with infant occupancy assumed to track that of the parents.

Different module combinations can be used to replicate actual household types. For example, a family household with one adult child and one under- 16 child combines a parent module, a single-person module, and a child module linked to the parent model output.

\subsubsection{Day Types and Occupant Calendars}

To allow the model to replicate the behaviour of real households, each occupant is defined by age and working or education status. Separate transition probability matrices (TPMs) have been generated for each defined age range, for each day type (weekday, Saturday and Sunday), and whether the occupant is working, not working or in education. For couples and parents, there are three options; both working, one working and both not working. Workers are allocated typical working weeks 
based on analysis of the separate one-week working diaries in the UK 2000/1 Time Use Survey dataset [11].

Individual calendars are then defined for each individual to reflect the sequence of day types through the modelling period. The model selects the appropriate TPM for the required day type as necessary. The model can therefore clearly distinguish different typical occupancy behaviours for each occupant types (full-time workers, stay-at-home parents, students, school children etc.), that is a key precursor to demand prediction for each occupant and household type.

\subsection{Model Output Assessment}

The primary output from the model is a per-time step sequence of occupant states. While the validation metrics used allow the differences between profiles to be quantified, critical analysis of actual profiles can also demonstrate model robustness.

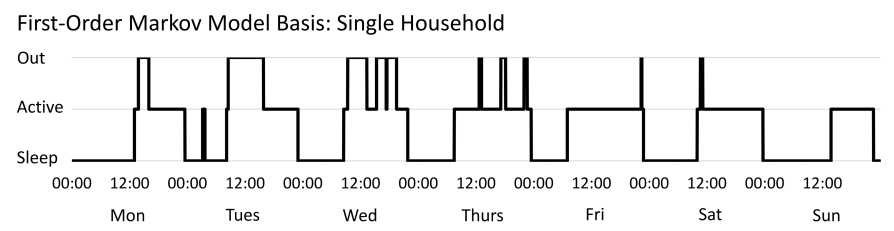

Higher-Order Markov Model: Single Household, 33-40 yo, Working Mon-Sat

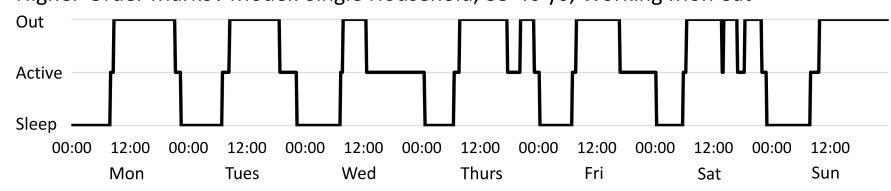

Higher-Order Markov Model: Single Household, 80+, Retired

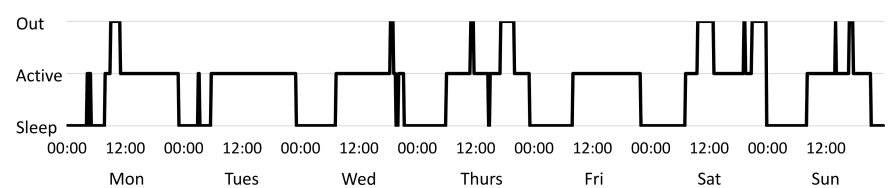

Fig. 11. Example one-week individual occupancy state profiles for various modelled one-person households.

Figure 11 shows results from randomly selected model runs. The results compare a Mon-Sun sequence for a 1-person household using a first-order Markov-Chain approach (similar to Richardson et al [13]) with a younger working and older retired 1-person household using the developed differentiated higherorder Markov-Chain model. These populations were deliberately selected for a strong likelihood for longer out and active periods respectively.

The first-order, larger population model shows no overall consistency between modelled days. This gives credence to the assertion that this type of model generates profiles that are an unrepresentative composite of multiple behaviours.

The developed higher-order model more consistently models sleep durations within the most likely duration range, shows daily out periods consistent with a working person, and long active periods consistent with an older retired person.

For multi-person households the model generates individual outputs as per Figure 11 and also consolidated profiles of total adult, child and overall occupant number per timestep. Figure 12 shows an example output for a two-adult/two-child family with one full-time worker for a typical school term week. The modelled link between adult and child occupancy in particular is highlighted.

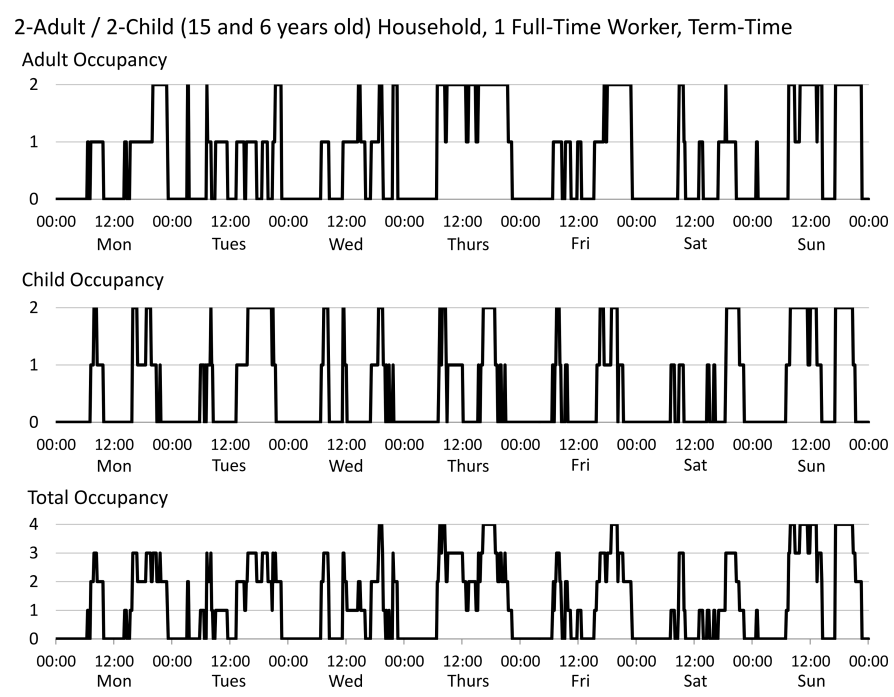

Fig. 12. Example one-week occupant number profiles for a modelled 2-adult/2child household.

\subsection{Model Applications and Limitations}

Any TUS-based model has inherent limitations as a result of the 24-hour basis of the diaries used to generate the calibration data. For multiple annual model runs, the modelling method identified does provide some degree of variability in overall average active occupancy (e.g. +/- c.10\% for 'Working 18-37' models) but it is not possible to assess if this overall result or the specific sequence of days is realistic. As a minimum, it is unlikely that the model captures extreme levels of occupancy within populations and definitely does not model households with repetitive behaviours (e.g. same weekday wake time, fixed work hours etc.).

However, the analysis presented here and by others ([2],[18]) has clearly demonstrated that there are broad occupancy patterns related to identifiable household types, and that existing models have underused the available data at this level of differentiation.

This model has been developed primarily to generate input occupancy data for a high time resolution, occupancy-driven energy model with the aim to identify specific demand patterns for homogenous communities (e.g. retirement, social housing, commuter) when compared to nationally representative populations and the specific influence of occupancy on any identified differences. At this resolution, the impact of averaging and inaccuracies associated with individual profiles will be reduced. For analysis of individual households, the model has some applicability but with significant qualification.

\section{Discussion and Conclusion}

Several enhancements to existing high resolution, occupancy models have been considered, with three primary potential improvements implemented and analysed. The first was to split 
the occupant models based on occupant and household characteristics, and different day types. These are combined based on realistic sequences of day types to generate more representative occupancy profiles that reflect different lifestyles. The second potentially improves the model consistency and status duration prediction by using a higher-order Markov-Chain process. The third was to differentiate between single, couple and family households, modelling couple and parent pairs as single entities to capture occupancy interactions.

Three metric were identified to allow model performance to be quantified. Analysis of the model output has shown significant improvement associated with both highly differentiated occupant models and using different methods based on household type. The higher-order method has been shown to be an improvement on existing methods, however the benefit is less significant to overall modelling accuracy. Further analysis with new occupancy validation data, and also of relative demand model prediction accuracy, using both first- and higher-order approaches will be required to determine the resolution and scenarios for which the higher-order approach is beneficial and circumstances when the simpler first-order approach may suffice.

Previous modelling work in this area has focused on maintaining large, statistically robust populations to ensure model stability at the expense of combining data from groups with highly variable occupancy behaviours. Analysis of the available occupancy data highlighted that household type, age and employment status on the specific day of interest were all key determinants of occupancy behaviour. The presented work has demonstrated that the model remains stable for smaller populations down to 200 sets of single-day data. This has allow the occupancy data used to calibrate the model to be split by key identified criteria to capture typical behavioural differences. It has also allowed for the development of the single entity models for couples and parents from the limited existing occupancy data.

The Markov-Chain approach has been shown to remain an effective method for stochastic occupancy modelling from currently available data. A more computationally efficient 'event' method was reviewed but shown to perform poorly in the key occupancy transition periods. The use of higher-order models was shown to be more effective for input dataset replication but will require significantly more input and validation data for the full benefit to general occupancy prediction to be determined.

The model output remains limited by the lack of large, multiday occupancy datasets. The output is not suitable for detailed analysis at the single household level as it remains a composite of a wide range of behaviours. However, it provides the means to identify key occupancy variations between different household types, and potentially the influence on energy demand patterns, with a particular relevance for microgeneration schemes for communities (i.e. $<500$ households) with distinct household type distributions. The developed model also seeks to move beyond traditional occupant archetype methods and to identify individuals by a more realistic and personalised sequence of different day types that reflect actual lifestyles.

Further major improvements in domestic occupancy prediction using this or other methods will require significantly better data combining the number and representative range of households typically analysed in Time-Use Surveys with simpler occupancy related states (i.e. wake, first leave, last return, sleep etc.) logged over longer periods.

\section{Acknowledgements}

We gratefully acknowledge the financial support received for this work from the BRE Trust.

\section{References}

[1] Abu-Sharkh, S., Arnold, R., Kohler, J., Li, R., Markvart, T., Ross, J., Steemers, K., Wilson, P., Yao, R., 2006. Can microgrids make a major contribution to uk energy supply? Renewable and Sustainable Energy Reviews 10 (2), 78-127.

[2] Aerts, D., Minnen, J., Glorieux, I., Wouters, I., Descamps, F., 2014. A method for the identification and modelling of realistic domestic occupancy sequences for building energy demand simulations and peer comparison. Building and environment 75, 67-78.

[3] Capasso, A., Grattieri, W., Lamedica, R., Prudenzi, A., 1994. A bottomup approach to residential load modeling. Power Systems, IEEE Transactions on 9 (2), 957-964.

[4] CTUR, 2015. [Accessed on 2nd February 2015]. URL https : //www.timeuse.org/node/4514.

[5] DECC, 2014. Community energy strategy: Full report. A report by the Department for Energy and Climate Change. January 2014

URL https://www.gov.uk/government/uploads/system/ uploads/attachment_data/file/275163/20140126Community_ Energy_Strategy.pdf

[6] Grandjean, A., Adnot, J., Binet, G., 2012. A review and an analysis of the residential electric load curve models. Renewable and Sustainable Energy Reviews 16 (9), 6539-6565.

[7] Haldi, F., Robinson, D., 2011. The impact of occupants' behaviour on building energy demand. Journal of Building Performance Simulation 4 (4), 323-338.

[8] McLoughlin, F., Duffy, A., Conlon, M., 2012. Characterising domestic electricity consumption patterns by dwelling and occupant socioeconomic variables: An irish case study. Energy and Buildings 48, 240248.

[9] Muratori, M., Roberts, M. C., Sioshansi, R., Marano, V., Rizzoni, G., 2013. A highly resolved modeling technique to simulate residential power demand. Applied Energy 107, 465-473.

[10] Nijhuis, M., Gibescu, M., Cobben, J., 2016. Bottom-up markov chain monte carlo approach for scenario based residential load modelling with publicly available data. Energy and Buildings 112, 121-129.

[11] ONS, 2003. United kingdom time use survey,2000. Downloaded from UK Data Service. 9th September 2003 (3rd) edition.

[12] ONS, 2007. Ons omnibus survey, time use module, february, june, september and november 2005. Downloaded from UK Data Service. 27th June 2007 edition.

[13] Richardson, I., Thomson, M., Infield, D., 2008. A high-resolution domestic building occupancy model for energy demand simulations. Energy and buildings 40 (8), 1560-1566.

[14] Rubner, Y., Tomasi, C., Guibas, L. J., 2000. The earth mover's distance as a metric for image retrieval. International journal of computer vision 40 (2), 99-121.

[15] Torriti, J., 2012. Price-based demand side management: Assessing the impacts of time-of-use tariffs on residential electricity demand and peak shifting in northern italy. Energy 44 (1), 576-583.

[16] Torriti, J., 2014. A review of time use models of residential electricity demand. Renewable and Sustainable Energy Reviews 37, 265-272.

[17] Widén, J., Nilsson, A. M., Wäckelgård, E., 2009. A combined markovchain and bottom-up approach to modelling of domestic lighting demand. Energy and Buildings 41 (10), 1001-1012.

[18] Wilke, U., 2013. Probabilistic bottom-up modelling of occupancy and activities to predict electricity demand in residential buildings. Ph.D. thesis, École Polytechnique Fédérale de Lausanne.

[19] Yao, R., Steemers, K., 2005. A method of formulating energy load profile for domestic buildings in the uk. Energy and Buildings 37 (6), 663-671. 
[20] Yohanis, Y. G., Mondol, J. D., Wright, A., Norton, B., 2008. Real-life energy use in the uk: How occupancy and dwelling characteristics affect domestic electricity use. Energy and Buildings 40 (6), 1053-1059. 